\title{
A REVIEW OF VIBRATION MACHINE DIAGNOSTICS BY USING ARTIFICIAL INTELLIGENCE METHODS
}

\section{Grover Zurita, Vinicio Sánchez y Diego Cabrera}

\begin{abstract}
In the industry, gears and rolling bearings failures are one of the foremost causes of breakdown in rotating machines, reducing availability time of the production and resulting in costly systems downtime. Therefore, there are growing demands for vibration condition based monitoring of gears and bearings, and any method in order to improve the effectiveness, reliability, and accuracy of the bearing faults diagnosis ought to be evaluated. In order to perform machine diagnosis efficiently, researchers have extensively investigated different advanced digital signal processing techniques and artificial intelligence methods to accurately extract fault characteristics from vibration signals. The main goal of this article is to present the state-of-the-art development in vibration analysis for machine diagnosis based on artificial intelligence methods.
\end{abstract}

Keywords: Artificial Intelligence Method, Machine Learning Method, Random Forest, Deep Learning. 